\title{
Philonsorbonne
}

11 | 2017

Année 2016-2017

\section{Performativité du langage et empowerment féministe}

Mona GÉRARDIN-LAVERGE

\section{OpenEdition}

1 Journals

Édition électronique

URL : https://journals.openedition.org/philonsorbonne/917

DOI : 10.4000/philonsorbonne.917

ISSN : $2270-7336$

Éditeur

Publications de la Sorbonne

\section{Édition imprimée}

Date de publication : 1 janvier 2017

Pagination : 93-105

ISSN : 1255-183X

\section{Référence électronique}

Mona GÉRARDIN-LAVERGE, «Performativité du langage et empowerment féministe », Philonsorbonne [En ligne], 11 | 2017, mis en ligne le 16 janvier 2017, consulté le 09 juin 2021. URL : http:// journals.openedition.org/philonsorbonne/917 ; DOI : https://doi.org/10.4000/philonsorbonne.917

\section{(c) Tous droits réservés}




\title{
Performativité du langage et empowerment féministe
}

\author{
Mona GÉRARDIN-LAVERGE
}

\section{Introduction}

«Un enfant de treize ans est en voiture avec son père quand ils ont un accident. L'ambulance vient les chercher, et le père meurt pendant le trajet. L'enfant est transporté à l'hôpital. Le meilleur médecin de l'hôpital entre dans la salle d'opération, voit l'enfant et s'exclame : "Mais je ne peux pas l'opérer, c'est mon fils !" Comment est-ce possible ? ».

Cette énigme bien connue a été posée à de nombreuses personnes lors d'un microtrottoir réalisé par l'Institut EgaliGone dans les rues de Lyon en $2013^{1}$. Rares sont les personnes qui vont imaginer que le médecin peut être la mère du garçon. Cet exemple a été utilisé pour défendre la féminisation des noms de métier, parce qu'il montre que la prétendue neutralité et universalité du genre masculin, qui ne marquerait pas nécessairement le masculin mais pourrait jouer le rôle de générique neutre (humain), n'est pas vraie. Quand on utilise le masculin, on véhicule et on produit généralement une représentation qui est celle d'un être humain genré. On pourrait dire que cela ne vaut que dans le cas où nos représentations sont déjà sexistes et où ce n'est pas le langage qui forge cette représentation. Je propose au contraire de penser que le langage participe à construire les stéréotypes sexistes qu'il semble simplement refléter, d'une multitude de manières : par l'existence de certaines catégories et de certains concepts ${ }^{2}$, par les nominations, les

1. https://www.youtube.com/watch?v=YebfaWkng9s

2. Comme l'explique Christine Delphy, les concepts dont nous disposons pour nommer et classer les groupes humains sont toujours des hiérarchisations et des instruments de domination. Les classifications sociales posent toujours un groupe qui constitue la norme et le groupe qui est « différent»: Christine DelPHY, Classer, dominer - Qui sont les «autres »?, Paris, La Fabrique, 2008. 
adresses et les injures, par la place accordée aux personnes dans une interaction langagière ${ }^{3}$.

Il semble donc nécessaire de proposer une analyse féministe de ce que nous fait le langage, et de ce « je » ou ce «nous » à qui le langage « fait» des choses. Cette nécessité se décline en trois questions : Quelle est la force, le pouvoir du langage, que construit-il pour nous, en nous ? Quelle peut être notre capacité de résistance aux catégories oppressives, à l'hétérosexisme véhiculé et produit par le langage ? Quel est ce « je » ou ce « nous » qui est construit par le langage et qui, en même temps, peut lui résister?

C'est dans le cadre de ce questionnement féministe anti-essentialiste sur le langage que je commencerai par exposer la représentation du langage sur laquelle on peut s'appuyer pour comprendre son rôle dans la construction des sexes, des genres et des sexualités : la représentation du langage comme performatif (1). Mais nous verrons que cette idée du langage pose problème, dans la mesure où elle fonde l'efficacité du langage sur l'autorité sociale détenue par les locuteurs et les locutrices ${ }^{4}$, elle-même déterminée par des structures sociales inégalitaires, donc inégalement répartie. Ainsi, nous avons besoin de comprendre ce qui est fait dans et par le langage, mais il est difficile de savoir si l'on peut faire autre chose avec les mots qu'entériner les rapports sociaux de domination, donc si l'on peut construire une puissance d'agir et un empowerment féministes dans le langage. C'est cette question que je vais tenter d'affronter dans cet article, en proposant une conception de la performativité du langage qui permette de penser la possibilité, pour des locuteurs et des locutrices non autorisé-es, de réaliser des actes de langage et, ainsi, de fragiliser l'ordre social inégalitaire qui nie leur droit à parler et leur impose le silence (2).

\section{Que fait le langage?}

Le langage « ordinaire » est le langage que l'on parle tous les jours, le langage réel, par opposition au langage philosophique, technique, scientifique, ou logique ${ }^{5}$. Pour J. L. Austin, le langage ordinaire n'est pas

3. Cf. Corinne Monnet, «La répartition des tâches entre les femmes et les hommes dans le travail de la conversation », publié sur le site « Les Mots Sont Importants » en janvier 2008.

4. Convaincue du rôle du langage dans la construction du genre et de l'oppression sociale et politique, je reprends et pratique des formes d'exploration linguistiques et graphiques féministes, qui cherchent à visibiliser les rapports de pouvoir constitutifs de la langue et à déconstruire la prétendue naturalité et nécessité du binarisme de genre. J'emploie habituellement des formes hétérogènes et exploratoires pour mettre en évidence le rôle du langage dans la bicatégorisation de genre, comme " illes », " locuteurices », qui ne visent pas à "représenter les femmes dans la langue », mais au contraire à contester les catégories de « femmes » et d' « hommes ». À la demande de la revue, j'ai employé ici une féminisation plus traditionnelle.

5. Sandra LAugier, Du réel à l'ordinaire, Quelle philosophie du langage aujourd'hui ?, Paris, Vrin, 1999. 
un outil neutre qui ne ferait que traduire fidèlement d'un côté le monde, les choses, et de l'autre la pensée de la personne qui s'exprime ${ }^{6}$. Le langage n'est pas un simple reflet, une simple représentation du monde, qui ne serait en connexion avec le monde que pour parler de ce dernier. Les énoncés ne sont donc pas seulement susceptibles d'être vrais (s'ils correspondent à l'état du monde) ou faux (s'ils ne lui correspondent pas). Bien au contraire, les énoncés sont des actes de parole, qui font des choses, qui agissent dans le monde. Ils sont performatifs, ce qui signifie qu'ils produisent des effets, qu'ils peuvent échouer et qu'ils engagent une certaine forme de responsabilité des locuteurs et locutrices. Ainsi, même s'il peut sembler qu'une assertion se contente d'énoncer un état du monde, il est possible qu'elle soit en train de réaliser ce qu'elle dit: si je dis que " je lègue ma montre à mon frère » dans un testament, je ne me contente pas de le dire, je le fais. De même, c'est en disant que «La séance est ouverte » que la présidente d'un tribunal ouvre effectivement la séance ${ }^{7}$. Dans les exemples précédents, l'énoncé n'échoue pas s'il est faux mais s'il ne parvient pas à faire ce qu'il cherche à faire. Par exemple, si je vais dans un tribunal et que je dis "la séance est ouverte», on va me rire au nez, et le procès ne commencera pas. Mon énoncé échouera comme performatif, parce que je ne suis pas juge et que je n'ai donc pas le statut social qui m'autorise à ouvrir la séance. Il y a des normes, des conventions sociales, qui définissent la performativité : il faut un certain statut social pour faire certaines choses avec certains mots.

Austin radicalise sa découverte du performatif et va plus loin que sa distinction entre constatif ou descriptif («le ciel est bleu») et performatif ( "la séance est ouverte»). Il explique que tout énoncé peut faire quelque chose en trois sens : déjà, tout énoncé est un acte de dire (au lieu de se taire) et, en cela, il a une fonction locutoire. L'énoncé peut également faire une chose au sens où, lorsqu'il est proféré, une nouvelle réalité est créée. C'est ce qu'Austin appelle l'acte illocutoire : un acte qui est accompli en disant quelque chose. Par exemple, quand une présidente de séance dit « La séance est ouverte », elle ouvre la séance en disant que la séance est ouverte. Pour finir, l'énoncé peut provoquer des sentiments, des pensées et des actions chez ses auditeurs et auditrices. Ces conséquences, dans le monde et sur les personnes, ne sont pas nécessaires, elles peuvent être imprévues. C'est ce qu'Austin appelle l'acte perlocutoire. Les effets qu'il produit sont plus

\footnotetext{
6. Selon cette conception du langage, le langage parle du monde parce qu'il le représente : par exemple, il y a un état du monde dans lequel le ciel est bleu, et auquel les énoncés « le ciel est bleu », « the sky is blue », « il cielo è azzurro » réfèrent tous. Le seul problème possible, c'est que le langage représente mal le monde : par exemple, je dis « le ciel est bleu » alors qu'il fait gris. Donc le seul échec du langage est d'être faux par rapport au monde, et le fait qu'un énoncé faux soit prononcé ne change rien au monde (le ciel reste gris même si je dis qu'il est bleu). (Pour cette analyse et pour ma présentation de la performativité austinienne, je m'appuie sur le livre de Bruno AmBroise, Qu'est-ce qu'un acte de parole?, Paris, Vrin, 2008).

7. John Langshaw Austin, How to Do Things with Words, Oxford University Press, 1962. Trad. Gilles LANE, Quand dire, c'est faire, Paris, Seuil, 1970.
} 
contingents que ceux de l'acte illocutoire, ils dépendent du contexte et de la réception de l'interlocuteur ou l'interlocutrice.

Cette représentation du langage ordinaire permet de montrer le caractère performatif d'énoncés apparemment descriptifs, qui posent une norme alors qu'ils semblent décrire le réel ${ }^{8}$. Elle constitue un outil extrêmement précieux pour un féminisme non-naturaliste et non-essentialiste, puisque contrer la représentation du langage comme simple reflet ou représentation du réel permet de critiquer l'illusion de la naturalité des rapports sociaux et de la domination. En retour, essayer de penser une puissance d'agir féministe dans le langage ordinaire permet de discuter et de repenser la performativité du langage.

\section{Puissance d'agir féministe}

La question à laquelle je vais m'intéresser maintenant est celle de la puissance féministe d'agir par rapport : (a) aux actes de parole hétérosexistes (les discours qui à la fois posent la dualité des sexes, qui la définissent comme naturelle - il y a deux sexes et ils sont complémentaires - et qui posent l'infériorité ou la subordination d'un des sexes par rapport à l'autre); (b) aux structures sociales inégalitaires (l'hétéropatriarcat) qui donnent leur pouvoir aux actes de parole hétérosexistes, et qui sont, selon moi, construites et renforcées par ces mêmes actes de parole; (c) à la construction comme inférieures des personnes appartenant aux groupes minorisés (nous nous situons ici davantage au niveau de la construction et de la représentation de soi, de la reconnaissance sociale, et de l'empowerment par rapport au manque de reconnaissance sociale).

Je souhaite proposer des pistes pour penser cette puissance d'agir, d'un point de vue théorique, en revenant sur certaines critiques adressées à Judith Butler à propos de la possibilité d'une subversion des discours de haine, et d'un point de vue pratique, politique et féministe, en partant de contreattaques et de subversions existantes, en les analysant pour en comprendre la portée et les conditions de réussite, et pour en suggérer l'extension possible à d'autres occurrences et à d'autres circonstances.

Qu'est-ce qu'une puissance d'agir féministe du langage et dans le langage? Qu'est-ce qui la rend possible et quelles peuvent être ses conséquences sur les rapports sociaux de sexe? Qu'est-ce que cette

\footnotetext{
8. C'est l'exemple canonique de l'énoncé du médecin lors de l'échographie : «C'est une fille» ou "C'est un garçon ». Cet énoncé, qui prétend constater un fait biologique, est en réalité un acte performatif par lequel l'embryon est en même temps reconnu comme humain et catégorisé comme "garçon » ou "fille ». Il inaugure une série d'énoncés dans laquelle l'enfant va grandir et se construire, qui seront pensés comme la suite logique de ce premier « constat», et qui imposeront à l'enfant de correspondre au sexe qu'on lui a assigné à la naissance. «Un garçon ne pleure pas », « Tu es une jolie petite fille », etc.
} 
puissance d'agir change à l'autorité sociale qu'on n'a pas mais qu'on prend, à la manière dont on se représente et dont on se positionne dans les rapports sociaux?

Je vais essayer de montrer qu'il existe une co-constitution du langage comme acte et des conditions sociales qui permettent aux énoncés de réussir comme actes de parole. C'est-à-dire que si la réussite d'un acte de parole, dans sa dimension d'efficacité et dans sa dimension de rapport correct avec le monde, est déterminée par des conditions extra-linguistiques et par les structures sociales ${ }^{9}$, il me semble cependant indispensable de comprendre comment les actes de parole peuvent avoir le pouvoir d'entériner ou au contraire de déstabiliser les rapports sociaux inégalitaires.

\subsection{Vulnérabilité des sujets au langage}

Tout d'abord, rappelons la réponse que Judith Butler apporte à la question de la puissance d'agir féministe dans et par le langage, dans $L e$ pouvoir des mots. Dans ce texte, Butler analyse les discours de haine et les injures fondées sur une domination sociale structurelle. Elle part du principe que les personnes sont vulnérables au langage, et que les discours de haine peuvent avoir sur elles un pouvoir extrêmement important : les discours de haine ont le pouvoir de dénier aux sujets une reconnaissance sociale pleine et entière ; ils peuvent affecter la représentation que les personnes ont d'ellesmêmes, menacer leur vie et leur être même. Pour Butler, il faut prendre la mesure du pouvoir des mots, et notamment de leur aspect performatif, mais il ne faut pas penser les discours de haine comme « souverains », (produisant des sujets complètement détruits, subordonnés et incapables de résistance). Elle reprend la distinction austinienne entre illocutoire et perlocutoire, qui lui permet de dire que, si les discours de haine affectent bien évidemment les personnes, ce n'est pas sur un mode illocutoire mais perlocutoire, de sorte que la manière dont elles sont affectées n'est jamais totalement prévisible ni certaine. Comme le dit Austin, un juge devrait pouvoir décider, en entendant ce qui a été dit, quels actes locutoires et illocutoires ont été exécutés, mais non quels actes perlocutoires. En d'autres termes, il faut reconnaitre, pour Butler, ce qu'elle appelle la «temporalité ouverte des actes de discours $»^{10}$ : changer le contexte d'un discours de haine peut lui retirer de son pouvoir de me blesser, mais pas entièrement. Il y a une sensibilité du discours au contexte, mais elle n'est pas totale, de sorte que si les mots ont un pouvoir sur nous, on peut cependant résister à ce pouvoir, subvertir les discours, les resignifier. Cette analyse permet de penser une résistance aux discours hétérosexistes qui ne passe pas par la censure ou la législation (comme

\footnotetext{
9. Bruno AMBroise, « Judith Butler et la fabrique discursive du sexe », publié dans Raisons politiques $\mathrm{n}^{\circ} 12$, Presses de Sciences Po, 2003/4.

10. Judith Butler, Excitable Speech : A Politics of the Performative, New York, Routledge, 1997. Trad. Charlotte Nordmann, Le Pouvoir des Mots, Politique du performatif, Paris, Éd. Amsterdam, Paris 2004, p. 36.
} 
le propose Catharine MacKinnon par exemple ${ }^{11}$ ), mais par la lutte et l'empowerment individuel et collectif.

Cependant, la question de l'empowerment et celle de la subversion font difficulté : Butler explique en effet que la possibilité et le succès d'une subversion ne sont jamais absolument certains, et qu'ils peuvent échouer. Il semble donc important de réfléchir à cette question : y a-t-il des conditions qui rendent possibles la subversion des discours hétérosexistes ? Comment peut-on attaquer l'hétérosexisme dans et par le langage? Peut-on s'appuyer sur certaines contre-attaques, certaines subversions réussies, pour trouver des stratégies applicables à d'autres situations?

\subsection{Conditions sociales de la réussite d'un acte de parole}

La question des conditions de la subversion tient au fait que, comme nous l'avons déjà dit, Austin pense que la réussite comme l'échec du performatif dépendent de l'autorité sociale détenue par la personne qui parle. Si je ne suis pas autorisée à ouvrir un procès, je ne l'ouvrirai pas même si je dis que la séance est ouverte. Il peut ainsi sembler prioritaire de lutter contre l'ordre social inégalitaire empêchant certaines personnes de faire des choses avec les mots, et on pourrait penser que la lutte politique ne se situe pas au niveau du langage mais des conditions sociales qui déterminent son efficacité. Cela soulève pourtant deux questions : d'abord, ne faut-il pas reconnaitre que certaines prises de parole et certains actes de langage sont faits par des personnes qui n'y sont pas autorisées, qui ne sont pas reconnues comme légitimes pour parler, de sorte qu'elles font évènement, qu'elles opèrent une rupture au sein de l'ordre social inégalitaire? Ensuite, les actes de parole n'ont-ils pas un réel pouvoir constitutif sur les conditions sociales, de sorte que si la réussite d'un performatif dépend des structures sociales, les actes de parole ont en retour le pouvoir de les bouleverser?

Pour répondre à ces questions, il faut commencer par rappeler en quoi la réussite d'un performatif dépend de conditions sociales. Comme l'explique Austin, dans Quand dire c'est faire, un énoncé performatif ne peut être effectué avec bonheur que si les conditions suivantes sont remplies :

«(A.1) Il doit exister une procédure, reconnue par convention, dotée par convention d'un certain effet, et comprenant l'énoncé de certains mots par de certaines personnes dans de certaines circonstances.

De plus,

(A.2) il faut que, dans chaque cas, les personnes et circonstances particulières soient celles qui conviennent pour qu'on puisse invoquer la procédure en question.

11. Voir par exemple Catharine MACKInNON, Only words, Cambridge, Harvard University Press, 1993. Trad. Isabelle CroIX et Jacqueline LAHANA, Ce ne sont que des mots, Paris, Des femmes, 2007. 
(B.1) La procédure doit être exécutée par tous les participants, à la fois correctement et

(B.2) intégralement

( $Г .1)$ Lorsque la procédure - comme il arrive souvent - suppose chez ceux qui recourent à elle certaines pensées ou certains sentiments, lorsqu'elle doit provoquer par la suite un certain comportement de la part de l'un ou l'autre des participants, il faut que la personne qui prend part à la procédure (et par là l'invoque) ait, en fait, ces pensées ou sentiments, et que les participants aient l'intention d'adopter le comportement impliqué. De plus,

$(Г .2)$ ils doivent se comporter ainsi, en fait, par la suite $»^{12}$.

Selon Austin, les conditions A et B sont indispensables pour que l'acte de parole performatif ait lieu, contrairement aux conditions $\Gamma$ qui ne conditionnent pas la réalisation de l'acte, mais permettent de déterminer si on abuse ou non de la procédure (par manque de la sincérité requise, par exemple). Par conséquent, certaines conditions sociales sont indispensables à la réalisation des performatifs, et plus précisément des illocutoires : l'efficacité d'un acte de parole repose sur une procédure définie par convention, qui détermine à la fois qui peut effectuer cet acte de parole, avec quels énoncés et dans quels contextes. La légitimité et l'autorité sociales sont donc indispensables à la réalisation d'un acte de parole dans sa dimension illocutoire. Une certaine forme de reconnaissance sociale est ainsi nécessaire : il faut que la communauté linguistique, mes interlocuteurs et interlocutrices, reconnaissent à la fois la procédure que j'invoque et le fait qu'elle a été respectée pour que mon acte de parole soit effectué avec succès.

À partir de là, plusieurs questions se posent: Que se passe-t-il lorsque j'essaie d'effectuer un acte illocutoire sans y être autorisée par convention? Et que se passe-t-il précisément pour les personnes qui sont construites comme inférieures ou subordonnées par les actes illocutoires autorisés et légitimés socialement? D'abord, est-ce que leur reconnaissance est réellement indispensable ? Comme le dit Austin, « la question se pose de savoir jusqu'à quel point les actes peuvent être unilatéraux $»^{13}$. Ensuite, peuvent-elles accorder une pleine et entière reconnaissance à ce type d'actes qui, précisément, leur dénie la reconnaissance sociale, donc la légitimité à accorder ou non leur reconnaissance à un acte de parole ?

En posant cette question, je propose d'étendre ce que dit Austin des insultes à l'ensemble des discours qui posent une inégalité sociale, et notamment aux discours hétérosexistes. En effet, Austin explique qu'insulter quelqu'un'e est une procédure conventionnelle, parce qu' « en un sens nous ne pouvons pas ne pas comprendre la procédure que l'autre a l'intention d'invoquer», et en même temps, "nous n'entrons pas dans son jeu». En

12. J. L. Austin, trad. cit., p. 49.

13. J. L. Austin, trad. cit., p. 66. 
disant cela, il semble qu'Austin mette en évidence une certaine complexité de la reconnaissance, qu' on peut accentuer ainsi : lorsqu'un discours sexiste m'est tenu, je ne peux pas ne pas reconnaitre la procédure invoquée, et en même temps il y a une forme de contradiction à la reconnaitre, puisque la reconnaitre c'est admettre mon infériorité sociale, mon statut subordonné, donc mon illégitimité à dire si oui ou non j'accorde ma reconnaissance. C'est une forme de contradiction au cœur même de l'acte de parole oppressif : il exige que nous reconnaissions que nous ne sommes pas légitimes à reconnaitre quoi que ce soit. Mettre au jour cette contradiction performative est un premier pas dans la déstabilisation de la reconnaissance que l'on accorde à l'ordre social inégalitaire, et donc aux conventions qui organisent qui peut faire quoi avec les mots. Je vais maintenant analyser certains actes de parole susceptibles à la fois de produire un empowerment féministe dans et par le langage et d'avoir un effet sur les conditions sociales.

\subsection{Prise de parole, prise de pouvoir}

Pour commencer, je propose de penser qu'il peut y avoir un succès féministe dans les effets perlocutoires même si, et peut-être parce que, l'acte a échoué sur le plan illocutoire. Par exemple, je peux utiliser une procédure en y étant autorisée mais dans des circonstances inadaptées (conditions A2), et sans avoir réellement l'intention de produire l'effet illocutoire prévu par la procédure (conditions $\Gamma$ ). Ainsi, dans son manuel d'autodéfense féministe intitulé Non c'est non, Irene Zeilinger nous conseille, en cas d'agressions, de ne pas appeler «Au secours », mais « Au feu »:

«Appeler "au secours!" par exemple n'est pas une bonne idée, car les gens ne tourneront guère la tête. D'abord, on crie facilement au secours pour rigoler, et puis le secours à porter n'est pas dans leur intérêt. Par contre, "au feu !" est un cri qui attire plus d'attention. Parce que c'est moins commun et parce que le feu concerne tout le monde : même des gens lâches et indifférents doivent au moins vérifier si ça brûle chez eux ou chez le voisin. Et le fait que des fenêtres s'ouvrent, que des gens regardent, peut déjà décourager des agresseurs, car, en général, on n'aime pas trop avoir du public quand on a des mauvaises intentions $»^{14}$.

Les conditions sociales peuvent rendre l'appel « Au secours » inefficace sur les plans illocutoire et perlocutoire. Il est cependant possible d'obtenir l'effet recherché par cet acte de parole (être aidée) en criant " $\mathrm{Au}$ feu ». Si cet acte ne peut qu'échouer sur le plan illocutoire (vous n'avez pas prévenu qui que ce soit du fait qu'il y avait un feu puisqu'il n'y a pas de feu), il est cependant susceptible de produire les conséquences perlocutoires que vous

14. Irene ZeILINGer, Non c'est non, Petit manuel d'autodéfense à l'usage de toutes les femmes qui en ont marre de se faire emmerder sans rien dire, Paris, Zones, 2008 (http://www.editions-zones.fr/spip.php?page=lyberplayer\&id article=60). 
n'auriez pas pu obtenir avec votre appel «Au secours ». Ainsi, prendre acte de notre inégalité face à la possibilité de faire ou non des choses avec les mots ne doit pas nous conduire à penser qu'on ne peut rien faire avec et dans le langage, bien au contraire. Si on échoue à obtenir les effets que l'on recherche en invoquant les procédures socialement prévues (ce qui est une forme de réduction au silence), on peut essayer d'obtenir ces effets avec d'autres énoncés qui n'étaient pas prévus pour ça. On peut subvertir la procédure déterminant la performativité d'un énoncé, en échouant intentionnellement sur le plan illocutoire pour réussir sur le plan perlocutoire.

Concernant la subversion, une des questions qui me semblent particulièrement intéressantes est celle de la définition des mots. En effet, la question des mots qui existent et de leur sens est une question politique déterminante : comme le dit Christine Delphy, on peut souvent mesurer le pouvoir social au pouvoir de catégoriser et de nommer ${ }^{15}$. Avoir le pouvoir de nommer, c'est avoir le pouvoir de faire exister et de faire reconnaitre ce qui existe pour nous et, en même temps, c'est avoir le pouvoir de dissimuler que cela existe pour nous, et que la reconnaissance de cela par autrui peut être le résultat d'un rapport de force. On peut penser, par exemple, à la question du travail : il y a un enjeu féministe à parler de "travail domestique » et non de "tâches domestiques », pour faire reconnaitre d'abord que ce travail devrait être ou bien également réparti ou bien rémunéré, et ensuite que la distinction travail/tâches est dans son essence patriarcale (étant donné que ce n'est ni la personne ni la tâche qui justifie la différence qu'on fait entre tâche et travail, c'est donc l'existence d'une sphère domestique définie par l'exploitation patriarcale des femmes) $)^{16}$.

À partir de là, on peut aller plus loin et poser la question suivante : si un acte de parole a des conséquences perlocutoires imprévues et subversives, n'a-t-il pas un certain pouvoir de modifier les conditions sociales et le statut des personnes qui parlent, donc, en fait, sa propre efficacité illocutoire ?

En d'autres termes, quand je dis «Cette activité-là, c'est du travail», voici ce qui peut se passer: je n'ai pas l'autorité sociale nécessaire pour définir ce mot, donc pour réussir mon acte sur le plan illocutoire (je ne suis pas à l'Académie française, je n'écris pas de dictionnaire, je parle de mon expérience). Mais cet acte de définition raté peut avoir des conséquences perlocutoires : je conteste la définition dominante du travail, je propose un sens ouvert à l'expérience d'autrui, et je conteste l'ordre social qui me refuse la légitimité à définir des mots. En d'autres termes, je conteste la place qu'on m'a assignée dans l'ordre social et j'essaie de faire reconnaitre l'autorité qu'on me conteste.

15. Christine Delphy, Classer, dominer - Qui sont les « autres »?, Paris, La Fabrique, 2008. 16. Christine DelPhy, L'ennemi principal, tome I, «Économie politique du patriarcat », Paris, Syllepses « Nouvelles Questions Féministes », 2001. 


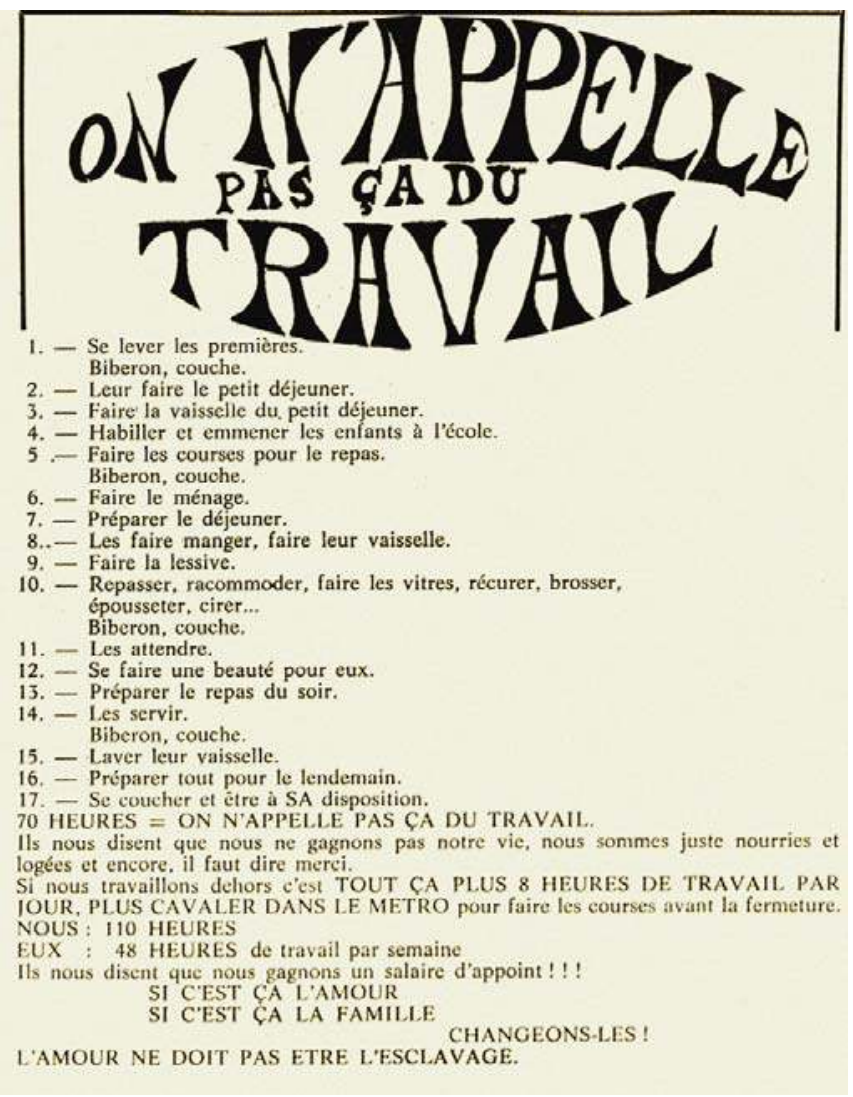

«On n'appelle pas çà du travail »

Article publié dans Le Torchon brûle, $\mathrm{n}^{\circ} 1,1971$, p. 8

Par exemple, dans l'image ci-dessus, extraite du journal Le Torchon brûle, journal féministe édité par le MLF (Mouvement de Libération des Femmes) dans les années 1970, on trouve une critique féministe de l'usage dominant du mot «travail » (celle que donne le dictionnaire, et qui est très importante à l'époque puisque, avec le marxisme, la question du travail est une question politique centrale). Cette contestation a pour objectif de faire reconnaitre le point de vue dominé, donc de changer les conditions sociales. Et en même temps elle se fait en refusant déjà les conditions sociales qui impliquent une non-reconnaissance de ce point de vue dominé : d'abord, par l'énumération des travaux domestiques réservés aux femmes, le texte pose les femmes comme autorité épistémique supérieure pour définir le travail (elles ont une expérience que les hommes n'ont pas, donc elles seules sont habilitées à dire si ce qu'elles font est ou non du travail). Ensuite, par l'appel à changer les conditions sociales, en critiquant les concepts traditionnellement utilisés pour imposer aux femmes de faire le travail domestique (l'amour et la famille) et, là encore, en en contestant le sens dominant. 
Ainsi, en disant que les tâches domestiques sont du «travail» je m'élève comme autorité faisant un travail et, alors que les conditions sociales devraient empêcher mon acte illocutoire de définition de réussir, il peut avoir un certain succès. Donc je reprends une procédure définie par convention mais je me l'approprie sans avoir l'autorité nécessaire. Bien sûr, on pourra nier mon autorité à recourir à cette procédure, et me rétorquer que «non ce n'est pas du travail». Mais il me semble que cette simple tentative produit au moins les deux effets suivants : (a) elle met en évidence le fait que la communauté linguistique n'est pas une communauté consensuelle, que les conventions sont le fruit de rapports de force et sont moins une source d'accord que de conflit; (b) en contestant l'ordre hiérarchique organisant qui parle et qui ne parle pas, elle peut produire un effet de resubjectivation des personnes et des groupes : comme l'explique Jacques Rancière, la prise de parole peut constituer une prise de pouvoir au double sens d'une contestation de l'ordre social inégalitaire et d'une resubjectivation des personnes dominées en personnes qui revendiquent leur égalité, leur autorité et leur légitimité à produire des actes de parole, donc à recourir à une procédure conventionnelle qui ne prévoit pas que ces mêmes personnes s'en emparent ${ }^{17}$. Il me semble que cette resubjectivation peut être saisie par les concepts de condition et de position proposés par Maria Puig de la Bellacasa ${ }^{18}$ : performer un acte de parole selon une procédure que l'on n'a pas la légitimité d'invoquer peut déstabiliser l'ordre social en transformant une condition sociale inégalitaire en position critique et de lutte.

Ces deux effets reposent sur la vulnérabilité des sujets et des processus de subjectivation au langage, qui à leur tour ont des conséquences sur les structures sociales. Il est ainsi possible qu'un acte de parole modifie les conditions sociales déterminant son efficacité illocutoire, ce qui implique de reconnaitre la possibilité d'une co-constitution de la performativité $\mathrm{du}$ langage et des conditions sociales.

\section{Conclusion}

Ma question principale était celle de la possibilité d'une puissance d'agir et d'un empowerment féministes dans et par le langage ordinaire, et plus précisément du rapport entre les conditions sociales et l'efficacité d'un acte de parole. En effet, si faire quelque chose avec les mots implique d'y être socialement autorisé $\cdot e$, plusieurs questions se posent: Qu'est-ce qu'être socialement autorisé e à faire quelque chose ? Existe-t-il quelque chose d'unitaire et de cohérent comme une communauté linguistique

17. Jacques RANCIERE, La Mésentente, Politique et philosophie, Paris, Galilée, 1995 ; Jacques RANCIERE, Aux bords du politique, Paris, Gallimard « Folio », 2004.

18. Maria Puig DE la Bellacasa, «Divergences solidaires », publié dans Multitudes $\mathrm{n}^{\circ} 12$, Printemps 2003, « Majeure 12. Féminismes, queer, multitudes ». 
qui détermine par convention la procédure selon laquelle un énoncé peut agir, ou ne faut-il pas au contraire admettre que la communauté linguistique est moins l'espace du consensus que du conflit, de sorte que tout acte de parole, c'est toujours avant tout la revendication de la légitimité qu'on a à l'effectuer, légitimité qui ne peut jamais être absolument certaine ? Si les conditions sociales déterminent la réussite d'un acte de parole, ne faut-il pas reconnaitre que les actes de parole ont un pouvoir, de confirmation ou de déstabilisation, de ces mêmes conditions sociales?

Pour répondre à ces questions, j'ai proposé des exemples d'actes de parole féministes, qui m'ont conduite à dire : d'abord qu'il est possible d'utiliser l'échec illocutoire d'un énoncé pour obtenir des effets perlocutoires qui subvertissent la procédure conventionnelle déterminant qui parle, de quoi et dans quelles circonstances. Ensuite, que si un acte de parole a des conséquences perlocutoires de contestation de l'hétérosexisme, il a alors un certain pouvoir de modifier les conditions sociales et le statut des locuteurs et des locutrices, donc, en fait, sa propre efficacité illocutoire. Pour finir, que l'une des conditions de ce processus est la vulnérabilité des sujets au langage, et notamment à leurs propres actes de parole et aux effets perlocutoires de ces actes sur la subjectivation individuelle et collective. De tels effets de resubjectivation produits par certains actes de parole ont des conséquences sur les conditions sociales ; on peut donc dire que si les actes de parole reposent sur des conditions sociales et sur des personnes dotées de statuts sociaux et possédant une connaissance des conventions sociales, les unes comme les autres peuvent être construites, modifiées, transformées dans et par le langage.

\section{Bibliographie}

Ambroise, Bruno, «Judith Butler et la fabrique discursive du sexe », publié dans Raisons politiques $\mathrm{n}^{\circ} 12$, Presses de Sciences Po 2003/4.

Ambroise, Bruno, Qu'est-ce qu'un acte de parole ?, Paris, Vrin, 2008.

Austin, John Langshaw, How to do things with words, Oxford University Press, 1962. Trad. Gilles Lane, Quand dire, c'est faire, Paris, Seuil, 1970.

Bernheim Cathy, Kandel Liliane, Picq Françoise, Ringart Nadja (coll.), MLF. Textes premiers, Paris, Stock, 2009.

Butler, Judith, Excitable Speech: A Politics of the Performative, New York, Routledge, 1997. Trad. Charlotte Nordmann, Le Pouvoir des Mots, Politique du performatif, Paris, éd. Amsterdam, 2004.

Delphy, Christine, L'ennemi principal, tome I, «Économie politique du patriarcat » et tome II, "Penser le genre », Paris, Syllepses « Nouvelles Questions Féministes », 2001, 2009. 
Delphy, Christine, Classer, dominer - Qui sont les «autres»?, Paris, La Fabrique, 2008.

Laugier, Sandra, Du réel à l'ordinaire, Quelle philosophie du langage aujourd'hui ?, Paris, Vrin, 1999.

MacKinnon, Catharine, Only Words, Cambridge, Harvard University Press, 1993. Trad. Isabelle Croix et Jacqueline Lahana, Ce ne sont que des mots, Paris, Des femmes, 2007.

Monnet, Corinne, «La répartition des tâches entre les femmes et les hommes dans le travail de la conversation», publié sur le site «Les Mots Sont Importants » en janvier 2008.

Puig de la Bellacasa, Maria, «Divergences solidaires », publié dans Multitudes $\mathrm{n}^{\circ} 12$, Printemps 2003, «Majeure 12. Féminismes, queer, multitudes ».

Rancière, Jacques, La Mésentente, Politique et philosophie, Paris, Galilée, 1995.

Rancière, Jacques, Aux bords du politique, Paris, Gallimard «Folio », 2004.

Zeilinger, Irène, Non c'est non. Petit manuel d'autodéfense à l'usage de toutes les femmes qui en ont marre de se faire emmerder sans rien dire, Paris, Zones, 2008. 